\title{
A phenotypic microarray analysis of a Streptococcus mutans liaS mutant
}

\begin{abstract}
Correspondence
Indranil Biswas

ibiswas@kumc.edu
\end{abstract}

Received 7 August 2008

Revised 22 September 2008

Accepted 27 September 2008
Jiaqin Zhang ${ }^{1,2}$ and Indranil Biswas ${ }^{1}$

\author{
${ }^{1}$ Department of Microbiology, University of Kansas Medical Center, Kansas City, KS 66160, USA \\ ${ }^{2}$ Department of Parasitology, Shandong University School of Medicine, 44\# Wenhua Xi Road, \\ Jinan, Shandong 250012, PR China
}

\section{INTRODUCTION}

The human pathogen Streptococcus mutans is a Grampositive bacterium with low $\mathrm{G}+\mathrm{C}$ content that resides in the oral cavity, and is considered to be the primary aetiological agent in the formation of dental caries (Loesche, 1986). This pathogen has evolved a biofilm lifestyle for survival in the dental plaque formed on the tooth surface (Hamada \& Slade, 1980). Strep. mutans is also an inducer of infective endocarditis, with more than $14 \%$ of viridians streptococcus-induced endocarditis caused by Strep. mutans (Hamada \& Slade, 1980; Loesche, 1986; Ullman et al., 1988). Strep. mutans uses sucrose, which is often present in the dietary carbohydrates, and converts it to sticky polysaccharides known as glucans (Banas \& Vickerman, 2003; Kuramitsu, 1993). With the help of surface-associated proteins that bind to glucan, Strep. mutans colonizes in the oral cavity through the formation of diverse, multispecies biofilms on the tooth surface, commonly known as dental plaque. This organism has developed several mechanisms to maintain its presence in the oral cavity (Carlsson \& Hamilton, 1994) and to withstand drastic environmental changes (Ahn et al., 2006; Lemos et al., 2005).

Adaptive responses of bacteria to environmental changes, such as nutrient limitation, oxygen deprivation, antibiotic

Abbreviations: IM-HK, intramembrane-sensing histidine kinase; PM, phenotypic microarray; TCS, two-component signal transduction system.

A supplementary table with full data from the PM analysis is available with the online version of this paper. stress and osmotic shock, are regulated by the so-called two-component signal transduction system (TCS) pathways (Dalton \& Scott, 2004; El-Sharoud, 2005; Mascher et al., 2006; Verneuil et al., 2004). TCSs typically consist of a membrane-bound sensor histidine kinase and a cytoplasmic response regulator, with a common biochemical mechanism involving phosphoryl-group transfer between two distinct protein components. The sensor histidine kinase is composed of two components: an amino-terminal sensor/input domain that detects specific stimuli from the extracellular environment, and a cytoplasmic transmitter/ histidine phosphotransferase domain that autophosphorylates at a specific histidine residue in response to stimulation of the sensor domain (Fabret et al., 1999; Mascher, 2006). The genome of Strep. mutans UA159 encodes a total of 14 TCSs (Biswas et al., 2008), in addition to an orphan response regulator, CovR (Ajdic et al., 2002; Biswas \& Biswas, 2006). These TCSs are critical for survival under adverse conditions, as well as for regulation of virulence-associated factors of this pathogen (Biswas et al., 2007, 2008; Biswas \& Biswas, 2006; Idone et al., 2003).

LiaSR is a TCS that is believed to be a part of a complex regulatory network that monitors and responds to cellenvelope stress in Bacillus subtilis (Gardete et al., 2006; Jordan et al., 2006). Homologous systems such as VraSR in Staphylococcus aureus (Gardete et al., 2006; Jordan et al., 2006) and CesSR in Lactococcus lactis (Martinez et al., 2007) have been shown to function in a similar manner. LiaS and VraS both belong to a subclass of intramembranesensing histidine kinases (IM-HKs) that are found exclusively in Gram-positive bacteria with a low G+C 
content (Firmicutes) (Mascher, 2006). Most IM-HKs sense various stresses to the cell envelope, and the gene targets of these TCSs are those that are involved in the maintenance of cell-envelope integrity, mediation of antibiotic resistance, or detoxification processes (Mascher, 2006). In Streptococcus pneumoniae, a LiaSR homologue, TCS03 (SP0386 and SP0387), was expressed as a part of the vancomycin stress response of this micro-organism (Haas et al., 2005).

LiaSR and VraSR are induced by various inhibitors of cellwall synthesis, but only VraSR has been shown to have a direct effect on the expression of genes related to peptidoglycan synthesis and antibiotic resistance (Butcher et al., 2007; Gardete et al., 2006). LiaSR is activated in the presence of sublethal concentrations of lipid II-interacting antibiotics, including bacitracin, vancomycin, ramoplanin and nicin, and strongly induces expression of its own locus (Mascher et al., 2003, 2004). LiaSR is also activated in the presence of cationic antimicrobial peptides, and, to a lesser extent, alkaline shock, detergents, ethanol, exposure to organic solvents, and secretion stress (Mascher, 2006). Additionally, it has been reported that LiaSR regulates the expression of the lia operon during the transition from exponential growth phase to stationary phase, in the absence of exogenous cell-wall inhibitors and in the presence of an as-yet-unidentified stimulus (Jordan et al., 2007). VraSR of Staph. aureus is also activated in the presence of cell-wall inhibiting antibiotics, such as $\beta$ lactams (meticillin, oxacillin, etc.) or glycopeptides (i.e. vancomycin); however, in contrast to LiaSR, activation of VraSR leads to the overexpression of a number of genes, including those associated with cell-wall biosynthesis and $\beta$-lactam antibiotic resistance (Gardete et al., 2006; Kuroda et al., 2003; Yin et al., 2006).

In Strep. mutans, the SMU.486/SMU.487 locus, which was formerly known as RR11/HK11 (Li et al., 2002), encodes a TCS that is homologous to LiaSR (Biswas et al., 2008). A recent study in our laboratory with the sensor kinase LiaS of Strep. mutans UA159 revealed several novel findings. It was found that LiaS negatively regulates expression of $g b p C$, which encodes a glucan-binding protein essential for biofilm formation and cariogenicity (Biswas et al., 2008; Chong et al., 2008). LiaS was also shown to regulate expression and secretion of mutacin, a bacteriocin produced by Strep. mutans to suppress the growth of other competitor bacteria present in the dental plaque (Chong et al., 2008). In another study, Li et al. (2002) showed that mutations in LiaS in Strep. mutans NG8 resulted in the formation of aberrant biofilms and an acid-sensitive phenotype. Therefore, LiaS appears to be very important for the pathogenesis of Strep. mutans.

To gain a complete understanding of the role of LiaS in Strep. mutans biology, physiological and phenotypic changes of a liaS mutant were compared to its isogenic parental strain using phenotypic microarray (PM) technology (Biswas \& Biswas, 2005; Bochner et al., 2001; Zhou et al., 2003). PM is an integrated system of cellular assays for the simultaneous, high-throughput screening of a large number of phenotypes. Here we report the results of PM analysis performed with a liaS mutant in which we examined nearly 2000 cellular phenotypes.

\section{METHODS}

Bacterial strains and growth conditions. Strep. mutans strains UA159 and IBS148 (Chong et al., 2008) were routinely grown in ToddHewitt medium (BBL; Becton Dickinson) supplemented with $0.2 \%$ yeast extract (THY) at $37{ }^{\circ} \mathrm{C}$. When necessary, spectinomycin (Sp, $300 \mu \mathrm{g}$ $\mathrm{ml}^{-1}$ ) was included in the growth medium. For some confirmatory growth experiments, Strep. mutans cultures were grown in THY containing $1 \% \mathrm{NaCl}, 3 \% \mathrm{Na}_{2} \mathrm{SO}_{4}$ or other chemicals as indicated.

PM analysis. PM analysis was performed using Biolog's PM service facility. A total of 20 96-well PM plates constituting eight metabolic panels (PM1 to PM8) and 12 sensitivity panels (PM9 to PM20) were used in this study. To assess the altered phenotypes of the liaS mutant (IBS148), the growth was compared to its parent Strep. mutans UA159 strain. The basic growth media and the conditions for PM analysis were published previously (Biswas \& Biswas, 2005; Bochner et al., 2001; Zhou et al., 2003). The inoculating cell densities used in this study were $1: 13$ dilution of $81 \%$ transmittance for both metabolic and sensitivity panels. PM analysis was conducted in duplicate after incubation of the strains at $37{ }^{\circ} \mathrm{C}$ for $72 \mathrm{~h}$. A mean height difference threshold of 50 for metabolic panels and a difference threshold of 60 for sensitivity panels were used to consider the difference between the two growths significant. The data were further confirmed by Student's $t$ test. The growth kinetics for UA159 was displayed as a red tracing, while IBS148 was displayed as a green tracing. The phenotypic changes listed in Table 1 were the changes detected in both PM runs (for a complete analysis, see Supplementary Table S1, available with the online version of this paper). Standard PM testing protocols are described in http:// www.biolog.com; the conditions are similar to those used here.

Antibiotic susceptibility stress. Disc inhibition assays were performed as described previously to evaluate antibiotic susceptibility of the Strep. mutans liaS mutant (Biswas et al., 2008). Antibiotic discs (6 $\mathrm{mm}$ in diameter; Becton Dickinson) were placed on THY agar plates inoculated with the wild-type or the liaS mutant strains. Following overnight incubation under microaerophilic conditions, the zones of inhibition were measured. In addition, susceptibilities to selected antimicrobial agents were tested using E-test strips (ABBiodisk) on THY agar plates.

\section{RESULTS}

The LiaSR family of TCSs is known to be involved in sensing cell-envelope stress. However, the nature of the cell-envelope stress and the genes under the control of the LiaSR regulon vary greatly depending on the organism. To better understand the role of LiaS in the cell-envelope stress response in Strep. mutans, we performed a complete PM analysis using a previously constructed liaS mutant strain, IBS148 (Chong et al., 2008). This strain was generated by replacing an internal part of the liaS ORF with an aad gene encoding spectinomycin resistance (Chong et al., 2008).

To ensure that the expression of the downstream liaR gene was unaffected in liaS mutant IBS148, we performed a 
Table 1. Growth advantages in the liaS mutant

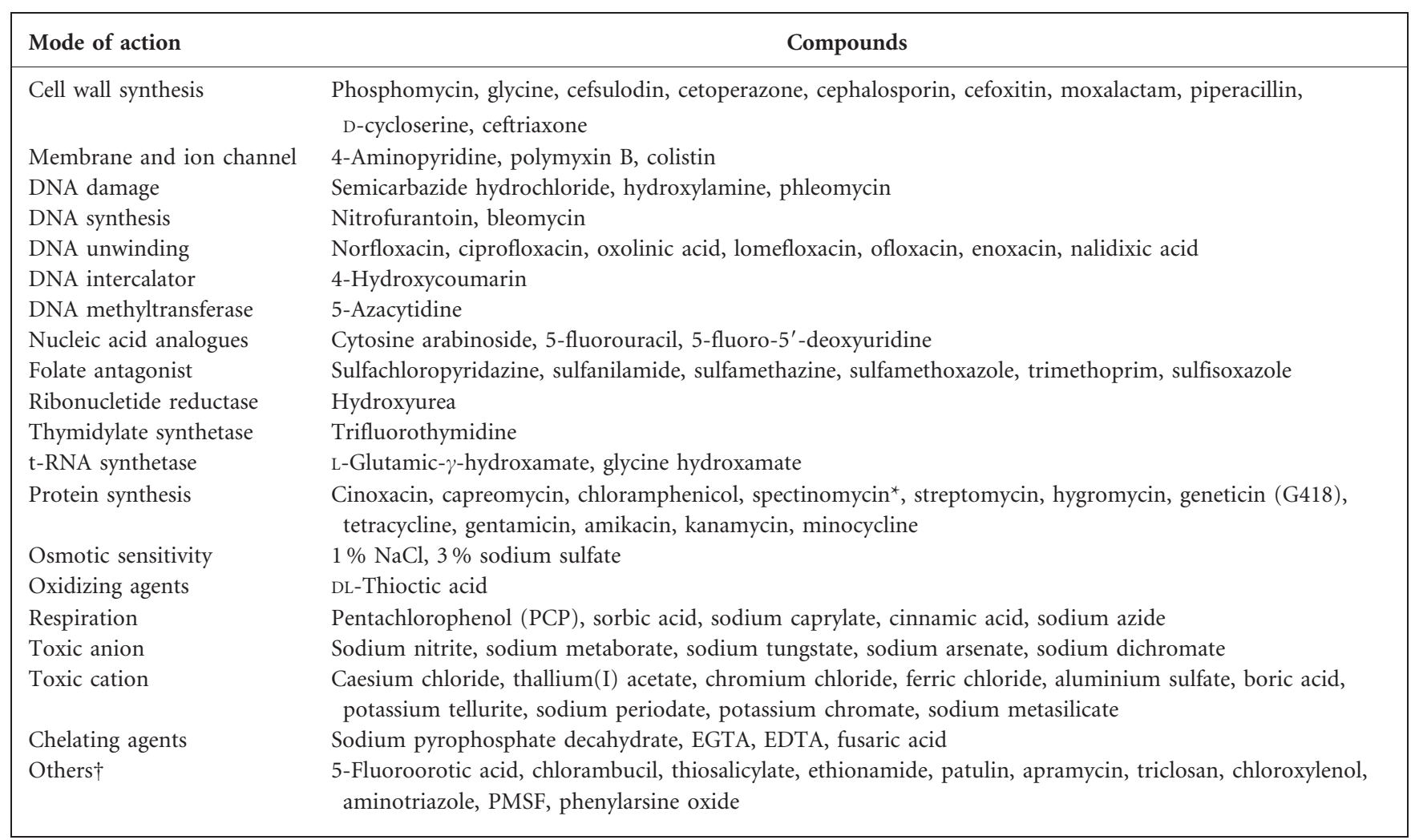

${ }^{\star}$ IBS148 contains a spectinomycin-resistant gene.

$\dagger$ Mode of action includes anti-capsule, antimicrobial, fatty-acid synthesis, histidine biosynthesis, tyrosine phosphatase.

semiquantitative RT-PCR analysis. RNA was extracted from strains UA159 and IBS148 after growth reached midexponential phase. Semiquantitative RT-PCR was performed using liaR-specific primers (Chong et al., 2008) to measure the level of liaR expression; the level of gyrA transcript was also measured to ensure that equal amounts of RNA were being used in the RT-PCR assay. As expected, the level of liaR transcript in IBS148 was equivalent to that of the wild-type strain UA159 (Fig. 1b), indicating that the insertion of the aad gene in liaS has little or no effect on the transcription of the downstream genes.

Since PM analysis relies on bacterial growth in media containing various growth inhibitors or toxic compounds, we wanted to measure the growth kinetics of IBS148 under nutrient-rich standard growth conditions. Both IBS148 and wild-type UA159 were grown in THY medium at $37{ }^{\circ} \mathrm{C}$ under static (microaerophilic) conditions. As shown in Fig. 1(c), the growth kinetics for both UA159 and IBS148 were very similar, indicating that there was no obvious growth defect in the liaS mutant.

PM analysis was first performed using the metabolic panels (PM1-8). There was no significant difference in the carbon utilization panels (PM1-2, data not shown). This was expected, since LiaS is not involved in sensing nutritional signals (Mascher, 2006; Mascher et al., 2006). The signals for the rest of the metabolic panels (PM3-8) were very low, which made the comparison difficult (data not shown). These panels include the nitrogen utilization panels (PM3, 6-7), phosphate and sulfate panel (PM4), and nutrient stimulation panel (PM5). The poor growth in these metabolic panels was not surprising since a previous study also demonstrated poor growth of a wild-type NG-8 strain, a different Strep. mutans strain from the one used in this study (Biswas \& Biswas, 2005).

PM analysis in the osmotic panel (PM10) generated mixed results (data not shown). In the majority of the wells, there were no growth advantages for either of the strains. However, UA159 produced stronger signals in media containing $4 \%$ urea and $0.2 \mathrm{M}$ sodium benzoate. In contrast, IBS148 generated stronger signals in media containing $1 \% \mathrm{NaCl}$ or $3 \%$ sodium sulfate. However, this growth advantage of IBS148 may not be significant since the growth kinetics of both IBS148 and UA159 were similar in THY medium supplemented with $1 \% \mathrm{NaCl}$ or $3 \%$ sodium sulfate (data not shown).

In the $\mathrm{pH}$ panel (PM9) there were no signals detected in about half the wells (data not shown). In the remaining wells, there were no significant differences between the wild-type and the liaS mutant strains. However, it was previously reported that a liaS mutant derivative of an NG- 
(a)
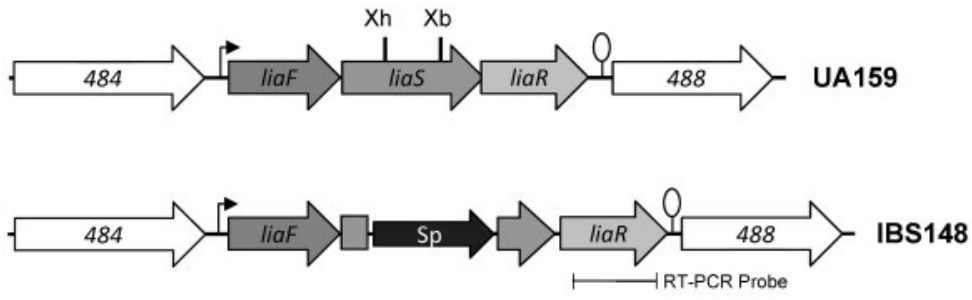

(b)

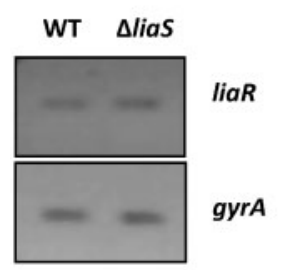

(c)

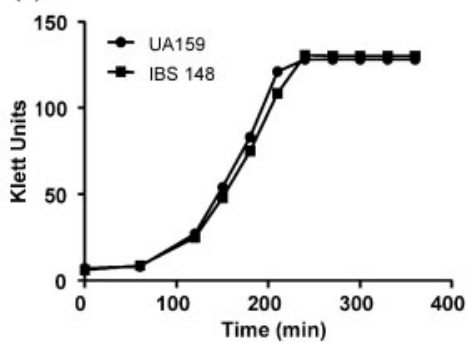

(d)

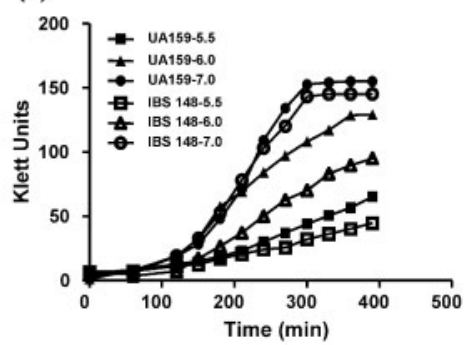

Fig. 1. (a) Schematic diagram of the lia locus in the wild-type UA159 and liaS mutant strains. Directions of transcription from the open reading frames are depicted by block arrows, with the bent arrow and the lollipop showing putative promoter and rhoindependent terminator, respectively. A part of the liaS ORF is deleted by $\mathrm{Xhol}(\mathrm{Xh})$ and $\mathrm{Xbal}(\mathrm{Xb})$ digestion and replaced with a spectinomycin-resistant gene aad (Sp). (b) Level of downstream liaR transcription measured by semiquantitative RT-PCR in the wild-type (UA159) and liaS mutant (IBS148). (c) Growth kinetics of UA159 and IBS148 in rich medium (THY). (d) Growth kinetics of UA159 and IBS148 in THY medium buffered with citrate/phosphate at the indicated pHs. Cultures were grown at $37{ }^{\circ} \mathrm{C}$ under microaerophilic conditions. Growth experiments were repeated at least three times and a representative growth curve is shown.

8 strain displayed a growth defect at $\mathrm{pH} 5.0$ ( $\mathrm{Li}$ et al., 2002). Under the PM condition tested, both UA159 and IBS148 grew equally well at $\mathrm{pH}$ 5.0, but they both failed to grow below pH 5.0 in PM plates. Failure to grow below pH 5.0 under the PM condition tested is not a strainspecific phenomenon, since a similar growth defect was previously reported for Strep. mutans NG-8 strain (Biswas \& Biswas, 2005). We have studied further the $\mathrm{pH}$ sensitivity of our IBS148 strain by growing the strain in THY medium buffered with citrate-phosphate buffer (Biswas et al., 2007). As shown in Fig. 1(d), IBS148 grew poorly compared to the wild-type UA159 in media with $\mathrm{pH}$ 6.0. At $\mathrm{pH}$ 5.5, both the strains grew poorly, whereas at $\mathrm{pH} 7.0$ both the strains grew equally well. Thus, taken together, our results show that LiaS is indeed involved in the acid-tolerance response of Strep. mutans. However, the apparent discrepancy between the growth of the cultures in the PM plate (at $\mathrm{pH}$ 5.0) and in the THY-buffer broths remain to be examined.

Surprisingly, PM analyses for the chemical sensitivity panels generated many positive differences for the IBS148 strains (Fig. 2). A list of the various inhibitors and toxic substances that produced a growth advantage for IBS148 is compiled in Table 1. IBS148 showed better growth than the wild-type UA159 in the presence of many antibiotics that target cell-wall biosynthesis, such as phosphomycin, Dcycloserine and cefoxitin (Fig. 2). A growth advantage was also observed for IBS148 in the presence of polymyxin B and colistin; both these chemicals target the cell membrane.
Compounds that also produced positive phenotypes for strain IBS148, but not the wild-type strain, include chemicals that block nucleotide biosynthesis, DNA replication or DNA unwinding, or induce DNA damage. Other chemicals such as folate antagonist, inhibitor of ribonucleotide reductase, and nucleotide analogues inhibited the growth of the wild-type UA159, but not the growth of IBS148. Similarly, IBS148 was resistant to many proteinsynthesis inhibitors, including chloramphenicol, gentamicin and tetracycline. Resistance to spectinomycin was also detected in IBS148 (Table 1), but this was presumably due to the presence of the aad gene used for the disruption of liaS. Resistance was also noted with chemicals that interfere with t-RNA synthesis, such as glycine hydroxamate and Lglutamic- $\gamma$-hydroxamate.

IBS148 displayed growth advantages in the presence of many toxic anions and cations, such as sodium nitrate, sodium arsenate and potassium tellurite, to name a few (Table 1). Several chelating agents, such as EGTA and EDTA, also generated positive results for IBS148. In addition, growth of IBS148 was better in the presence of chlorambucil, thiosalicylate, ethionamide, patulin, apramycin, triclosan, PMSF and phenylarsine oxide. Cellular targets for some of these compounds such as triclosan (fatty-acid biosynthesis) and PMSF (protease inhibitor) are known, while cellular targets of the other chemicals in Strep. mutans are currently unknown.

To confirm the PM sensitivity results, some of the key findings were investigated further by performing inde- 


\section{$\begin{array}{lll}\text { PM11 PM12 PM13 } & \end{array}$}

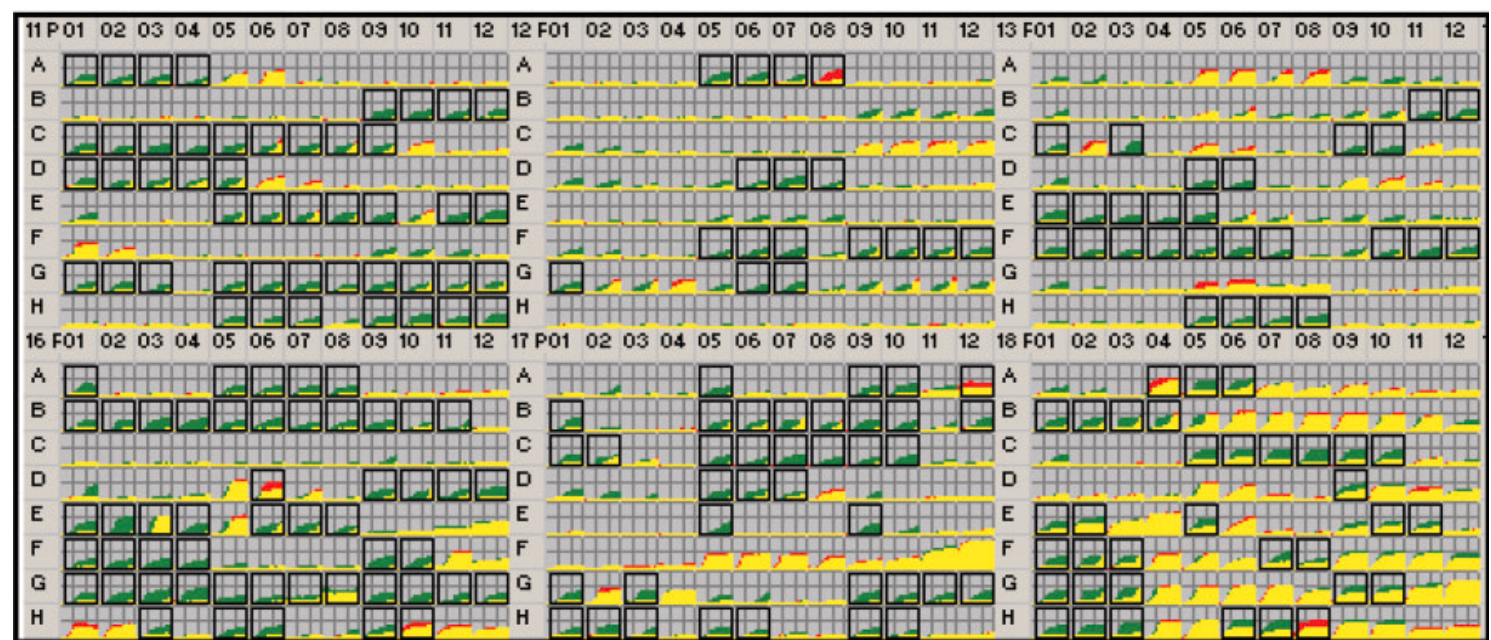

PM16

Fig. 2. $P M$ analysis for sensitivity to various antibiotics and toxic compounds. A complete catalogue of the reagents used in the sensitivity panel (PM11-PM20) is listed at http://www.biolog.com. The wild-type and the liaS mutant were grown in a 96-well plate under different conditions. Growth kinetics were obtained with the OmniLog instrument, a video-based detection system that detects colour development of tetrazolium dye due to bacterial respiration. Growth kinetics of wild-type and liaS mutant are superimposed using OmniLog software. The PM kinetic results show consensus data comparing the liaS mutant (IBS148, green) and its wild-type parental strain (UA159, red). A growth advantage by the parent is indicated by red, while a growth advantage by the liaS mutant is shown by green. When both strains have equal growth response or metabolisms in a well, the red and green kinetics overlap and produce a yellow colour. Two independent growth kinetic experiments were performed. A box around a well indicates a difference in response that was observed in both the experiments. The phenotypic changes are listed in Table 1; for a complete list, please refer to Supplementary Table S1.

pendent studies (Fig. 3). Susceptibilities to some antibiotics were verified using antibiotic disc diffusion assays and MICs were determined using E-tests. As shown in Fig. 3, treatment with phosphomycin (cell-wall inhibitor) or trimethoprim (folate antagonist) resulted in a visible difference in growth for IBS148 when compared with UA159. The MIC for ciprofloxacin (DNA gyrase inhibitor) was also twofold higher in IBS148 (MIC $2.0 \mu \mathrm{g} \mathrm{ml} \mathrm{g}^{-1}$ ) compared to UA159 (MIC $0.75 \mu \mathrm{g} \mathrm{ml}^{-1}$, data not shown). No difference in vancomycin resistance was observed between the UA159 and IBS148 strains (MIC $2.0 \mu \mathrm{g}$ $\mathrm{ml}^{-1}$ ). Taken together, our results demonstrate that IBS148 is more tolerant to several antibiotics that interfere with cell-wall biosynthesis and to compounds that interfere with the overall DNA replication process.

\section{DISCUSSION}

In B. subtilis and Staph. aureus, IM-HKs such as LiaS and other sensor kinases are involved in sensing cell-envelope stresses (Jordan et al., 2008; Mascher, 2006). Although the term 'cell-envelope stress' is not well defined, it generally indicates a condition that affects the composition and integrity of the cell membrane. These IM-HKs function as a sentinel system to detect conditions that presumably damage the integrity of the envelope or interfere with cell-wall synthesis. However, the nature of the signal that is detected by LiaS or its homologues can vary depending on the organisms. In B. subtilis, LiaS generally detects damage caused by antibiotics that interfere with lipid II recycling during cell-wall biosynthesis, such as bacitracin or vancomycin (Mascher et al., 2003, 2004); in Staph. aureus, VraS responds to an even broader spectrum of antibiotics, such as glycopeptides and $\beta$-lactams (Kuroda et al., 2003). In contrast to the wealth of knowledge on the role of LiaS in sensing cell-envelope stress in the above-mentioned organisms, little is known about its function in other Gram-positive pathogens, including Strep. mutans. In this study, we report a comprehensive phenotypic analysis of a liaS mutant of Strep. mutans where we document several unexpected findings.

Unlike its B. subtilis and Staph. aureus counterparts, inactivation of liaS in Strep. mutans produced gain-infunction phenotypes. For example, inactivation of $\mathrm{vraS}$ in Staph. aureus made the bacterium more susceptible to treatment with $\beta$-lactams and vancomycin (Kuroda et al., 
UA159

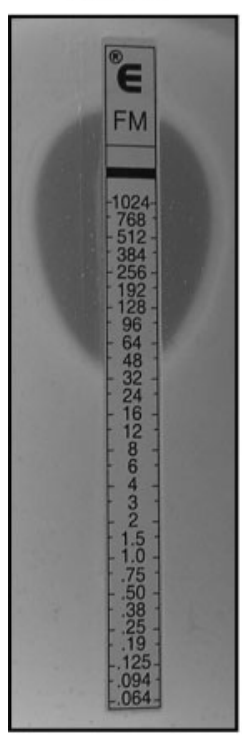

Phosphomycin
IBS148

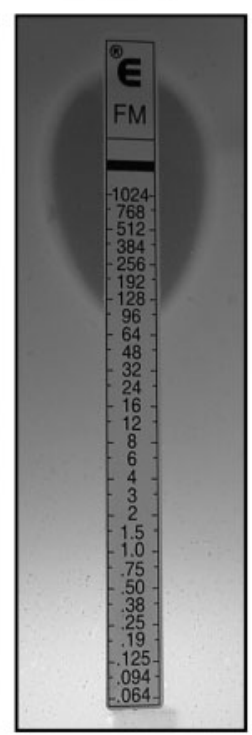$$
\text { in }
$$

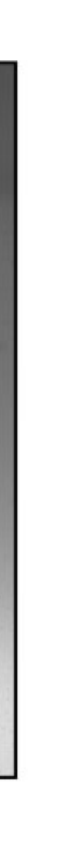

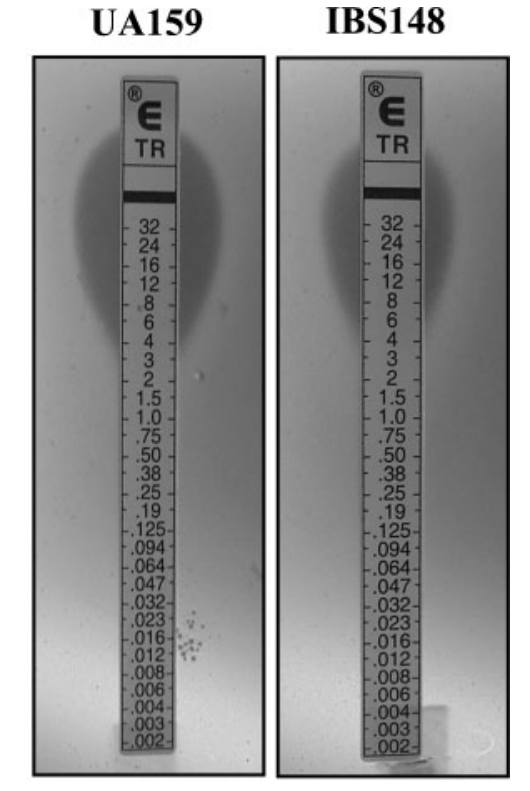

Trimethoprim
Fig. 3. Sensitivity to various antibiotics. E-strip tests were performed using either strain UA159 or strain IBS148 on THY agar plates. Plates were incubated at $37{ }^{\circ} \mathrm{C}$ under microaerophilic conditions for $20 \mathrm{~h}$.
2003). In contrast, we observed that deletion of the liaS gene in Strep. mutans resulted in increased tolerance, and not increased sensitivity, to cell-wall-damaging antibiotics such as phosphomycin and other $\beta$-lactams (Table 1 ). This is quite surprising since LiaS and VraS are positively involved in sensing cell-envelope stresses in B. subtilis and Staph. aureus, respectively (Mascher, 2006). One explanation could be that LiaS in Strep. mutans works differently than its counterpart in other bacteria. In Strep. mutans, it is possible that the primary function of LiaS is to inhibit the activity of LiaR, the cognate response regulator. In the absence of LiaS, LiaR might become constitutively activated (due to a change in phosphorylation status), allowing the gene expression necessary to survive cellenvelope stresses. The number and nature of the genes regulated by LiaR and its homologues differ greatly. In $B$. subtilis, LiaR regulates the expression of only two operons, including its own expression. On the other hand, there are approximately 46 genes in Staph. aureus and about 21 genes in L. lactis that are part of the LiaR regulon (Martinez et al., 2007). Negative regulation of LiaR by LiaS is rather possible in Strep. mutans, since a recent study shows that a liaR mutant is phenotypically quite similar to its isogenic wild-type parent, while a liaS mutant is not (Chong et al., 2008).

We also observed that two other antibiotics, vancomycin and bacitracin, which are known to be involved in LiaSdependent signalling, have no noticeable effect on the liaS mutant. In B. subtilis, there are at least three different IMHKs (LiaS, BceS and YvcQ) that are responsible for sensing different types of cell-envelope stresses. While LiaS can sense many different types of cell-envelope stresses, BceS only responds to bacitracin in B. subtilis (Jordan et al., 2008; Mascher, 2006). In Strep. mutans, MbrD
(SMU.1009), an IM-HK similar to LiaS, is involved in sensing cell-envelope stress generated by bacitracin (Tsuda et al., 2002). In addition, another sensor kinase, SMU.1965, also plays a role in bacitracin sensitivity (Biswas et al., 2008). Therefore, it is possible that either of these two sensor kinases, or both, may be involved in sensing cellenvelope stress generated by vancomycin, while LiaS takes part in detecting other cell-envelope stresses. However, based on the sequence homology and the genomic context, neither MbrD nor SMU.1965 appears to be the true homologue of LiaS.

We observed that the liaS mutant was more tolerant to compounds that interfere with nucleotide synthesis, DNA replication and DNA repair. This is rather surprising, since TCSs are not commonly involved in sensing DNA replication status or damage to DNA. It is possible that changes in the cell-envelope structure in the mutant strain may prevent these chemical agents from penetrating into the cell, or the efflux systems in the mutant are derepressed and the toxic chemicals are pumped out from the cell more efficiently, thereby making the liaS mutant more resistant to the action of these chemicals. On the other hand, there are a few examples in which TCSs have been shown to be involved in sensing DNA replication blockages or DNA damages. For example, in Caulobacter crescentus, a multicomponent signal transduction system involving three sensor kinases (CckA, PleC and DivJ), along with the master response regulator CtrA and another response regulator, DivK, respond to cell-cycle signals and other developmental cues (Jacobs-Wagner, 2004; Jenal, 2000). Moreover, in Escherichia coli, the ArcAB system seems to control initiation of DNA replication (Lee et al., 2001). Thus it is also possible that LiaS either directly or indirectly senses DNA replication blockage or DNA damage. 
In bacteria, the process of cell division and DNA replication is highly coordinated (Huisman \& D'Ari, 1981; Liu et al., 2001). Interruption of DNA replication or chromosomal segregation interferes with proper cell division, which ultimately leads to elongation of cells in rod-shaped bacteria such as E. coli and B. subtilis. However, in the case of Enterococcus faecalis, a spherical-shaped bacterium similar to Strep. mutans, inhibition of DNA synthesis by mitomycin C (Higgins et al., 1974) and nalidixic acid (Patel \& Weaver, 2006) leads to the inhibition of cell division, and an increase in cell-surface area. We speculate that treatment of Strep. mutans with chemicals that block DNA synthesis also leads to improper cell division that causes enlargement of the cell. This cell enlargement disrupts normal cell-envelope integrity, which is recognized by LiaS. Interestingly, a recent report indicates that in B. subtilis, a sensor kinase, YycG, coordinates cell-wall architecture during cell division by colocalizing with FtsZ at the cell-division septum and perceiving the signal (Fukushima et al., 2008). Strep. mutans also encodes a homologue of YycG, known as VicK; inactivation of vicK makes the Strep. mutans susceptible to many cell-wall-targeted antibiotics including $\beta$-lactams (Biswas et al., 2008). Whether LiaS or VicK also localize at a particular site on the cell surface for detecting cell-envelope stress signals remains to be examined in Strep. mutans.

The bacterial cell envelope is one of the crucial cellular structures whose integrity needs to be maintained at all times. Various environmental insults such as acidic $\mathrm{pH}$, high osmotic pressure and toxic chemicals, including antibiotics, can damage the integrity of the cell envelope. To cope with the cell-envelope damage, bacteria have evolved various mechanisms to detect perturbations to the envelope. There are at least four different types of signal transduction systems that sense and respond to cell-envelope stresses in Grampositive bacteria: TCSs; membrane-anchored anti- $\sigma$ factor and its corresponding ECF (extracytoplasmic function) $\sigma$ factor; transmembrane sensory protein BlaR1/MecR1; and a membrane-attached hybrid sensor/transcriptional regulator (Jordan et al., 2008). However, Strep. mutans does not encode any alternative $\sigma$ factors, and no BlaR1/MecR1 system has been identified in its genome. Therefore, we speculate that Strep. mutans primarily relies on TCSs to recognize cell-envelope stress, and LiaS is one of the most important IM-HKs that plays a significant role in recognizing cell-envelope stress in this pathogen.

\section{ACKNOWLEDGEMENTS}

We thank members of the Biswas laboratory for critically reading this manuscript. This work was supported in part by funding from NIDCR (DE016686) to I. B.

\section{REFERENCES}

Ahn, S. J., Wen, Z. T. \& Burne, R. A. (2006). Multilevel control of competence development and stress tolerance in Streptococcus mutans UA159. Infect Immun 74, 1631-1642.
Ajdic, D., McShan, W. M., McLaughlin, R. E., Savić, G., Chang, J., Carson, M. B., Primeaux, C., Tian, R., Kenton, S. \& other authors (2002). Genome sequence of Streptococcus mutans UA159, a cariogenic dental pathogen. Proc Natl Acad Sci U S A 99, 1443414439.

Banas, J. A. \& Vickerman, M. M. (2003). Glucan-binding proteins of the oral streptococci. Crit Rev Oral Biol Med 14, 89-99.

Biswas, S. \& Biswas, I. (2005). Role of HtrA in surface protein expression and biofilm formation by Streptococcus mutans. Infect Immun 73, 6923-6934.

Biswas, S. \& Biswas, I. (2006). Regulation of the glucosyltransferase $(g t f B C)$ operon by CovR in Streptococcus mutans. J Bacteriol 188, 988998.

Biswas, I., Drake, L. \& Biswas, S. (2007). Regulation of $g b p C$ expression in Streptococcus mutans. J Bacteriol 189, 6521-6531.

Biswas, I., Drake, L., Erkina, D. \& Biswas, S. (2008). Involvement of sensor kinases in the stress tolerance response of Streptococcus mutans. J Bacteriol 190, 68-77.

Bochner, B. R., Gadzinski, P. \& Panomitros, E. (2001). Phenotype microarrays for high-throughput phenotypic testing and assay of gene function. Genome Res 11, 1246-1255.

Butcher, B. G., Lin, Y. P. \& Helmann, J. D. (2007). The $y y d F G H I J$ operon of Bacillus subtilis encodes a peptide that induces the LiaRS two-component system. J Bacteriol 189, 8616-8625.

Carlsson, J. \& Hamilton, I. R. (1994). Metabolic Activity of Oral Bacteria, 2nd edn. Copenhagen: Munksgaard.

Chong, P., Drake, L. \& Biswas, I. (2008). LiaS regulates virulence factor expression in Streptococcus mutans. Infect Immun 76, 30933099.

Dalton, T. L. \& Scott, J. R. (2004). CovS inactivates CovR and is required for growth under conditions of general stress in Streptococcus pyogenes. J Bacteriol 186, 3928-3937.

El-Sharoud, W. M. (2005). Two-component signal transduction systems as key players in stress responses of lactic acid bacteria. Sci Prog 88, 203-228.

Fabret, C., Feher, V. A. \& Hoch, J. A. (1999). Two-component signal transduction in Bacillus subtilis: how one organism sees its world. $J$ Bacteriol 181, 1975-1983.

Fukushima, T., Szurmant, H., Kim, E. J., Perego, M. \& Hoch, J. A. (2008). A sensor histidine kinase co-ordinates cell wall architecture with cell division in Bacillus subtilis. Mol Microbiol 69, 621-632.

Gardete, S., Wu, S. W., Gill, S. \& Tomasz, A. (2006). Role of VraSR in antibiotic resistance and antibiotic-induced stress response in Staphylococcus aureus. Antimicrob Agents Chemother 50, 3424-3434.

Haas, W., Kaushal, D., Sublett, J., Obert, C. \& Tuomanen, E. I. (2005). Vancomycin stress response in a sensitive and a tolerant strain of Streptococcus pneumoniae. J Bacteriol 187, 8205-8210.

Hamada, S. \& Slade, H. D. (1980). Biology, immunology, and cariogenicity of Streptococcus mutans. Microbiol Rev 44, 331-384.

Higgins, M. L., Daneo-Moore, L., Boothby, D. \& Shockman, G. D. (1974). Effect of inhibition of deoxyribonucleic acid and protein synthesis on the direction of cell wall growth in Streptococcus faecalis. $J$ Bacteriol 118, 681-692.

Huisman, O. \& D'Ari, R. (1981). An inducible DNA replication-cell division coupling mechanism in E. coli. Nature 290, 797-799.

Idone, V., Brendtro, S., Gillespie, R., Kocaj, S., Peterson, E., Rendi, M., Warren, W., Michalek, S., Krastel, K. \& other authors (2003). Effect of an orphan response regulator on Streptococcus mutans sucrose-dependent adherence and cariogenesis. Infect Immun 71, 4351-4360. 
Jacobs-Wagner, C. (2004). Regulatory proteins with a sense of direction: cell cycle signalling network in Caulobacter. Mol Microbiol 51, 7-13.

Jenal, U. (2000). Signal transduction mechanisms in Caulobacter crescentus development and cell cycle control. FEMS Microbiol Rev 24, 177-191.

Jordan, S., Junker, A., Helmann, J. D. \& Mascher, T. (2006). Regulation of LiaRS-dependent gene expression in Bacillus subtilis: identification of inhibitor proteins, regulator binding sites, and target genes of a conserved cell envelope stress-sensing two-component system. J Bacteriol 188, 5153-5166.

Jordan, S., Rietkotter, E., Strauch, M. A., Kalamorz, F., Butcher, B. G., Helmann, J. D. \& Mascher, T. (2007). LiaRS-dependent gene expression is embedded in transition state regulation in Bacillus subtilis. Microbiology 153, 2530-2540.

Jordan, S., Hutchings, M. I. \& Mascher, T. (2008). Cell envelope stress response in Gram-positive bacteria. FEMS Microbiol Rev 32, 107-146.

Kuramitsu, H. K. (1993). Virulence factors of mutans streptococci: role of molecular genetics. Crit Rev Oral Biol Med 4, 159-176.

Kuroda, M., Kuroda, H., Oshima, T., Takeuchi, F., Mori, H. \& Hiramatsu, K. (2003). Two-component system VraSR positively modulates the regulation of cell-wall biosynthesis pathway in Staphylococcus aureus. Mol Microbiol 49, 807-821.

Lee, Y. S., Han, J. S., Jeon, Y. \& Hwang, D. S. (2001). The arc twocomponent signal transduction system inhibits in vitro Escherichia coli chromosomal initiation. J Biol Chem 276, 9917-9923.

Lemos, J. A., Abranches, J. \& Burne, R. A. (2005). Responses of cariogenic streptococci to environmental stresses. Curr Issues Mol Biol 7, 95-107.

Li, Y. H., Lau, P. C., Tang, N., Svensater, G., Ellen, R. P. \& Cvitkovitch, D. G. (2002). Novel two-component regulatory system involved in biofilm formation and acid resistance in Streptococcus mutans. J Bacteriol 184, 6333-6342.

Liu, G., Begg, K., Geddes, A. \& Donachie, W. D. (2001). Transcription of essential cell division genes is linked to chromosome replication in Escherichia coli. Mol Microbiol 40, 909-916.

Loesche, W. J. (1986). Role of Streptococcus mutans in human dental decay. Microbiol Rev 50, 353-380.

Martinez, B., Zomer, A. L., Rodriguez, A., Kok, J. \& Kuipers, O. P. (2007). Cell envelope stress induced by the bacteriocin Lcn972 is sensed by the lactococcal two-component system CesSR. Mol Microbiol 64, 473-486.

Mascher, T. (2006). Intramembrane-sensing histidine kinases: a new family of cell envelope stress sensors in Firmicutes bacteria. FEMS Microbiol Lett 264, 133-144.

Mascher, T., Margulis, N. G., Wang, T., Ye, R. W. \& Helmann, J. D. (2003). Cell wall stress responses in Bacillus subtilis: the regulatory network of the bacitracin stimulon. Mol Microbiol 50, 1591-1604.

Mascher, T., Zimmer, S. L., Smith, T. A. \& Helmann, J. D. (2004). Antibiotic-inducible promoter regulated by the cell envelope stresssensing two-component system LiaRS of Bacillus subtilis. Antimicrob Agents Chemother 48, 2888-2896.

Mascher, T., Helmann, J. D. \& Unden, G. (2006). Stimulus perception in bacterial signal-transducing histidine kinases. Microbiol Mol Biol Rev 70, 910-938.

Patel, S. \& Weaver, K. E. (2006). Addiction toxin Fst has unique effects on chromosome segregation and cell division in Enterococcus faecalis and Bacillus subtilis. J Bacteriol 188, 5374-5384.

Tsuda, H., Yamashita, Y., Shibata, Y., Nakano, Y. \& Koga, T. (2002). Genes involved in bacitracin resistance in Streptococcus mutans. Antimicrob Agents Chemother 46, 3756-3764.

Ullman, R. F., Miller, S. J., Strampfer, M. J. \& Cunha, B. A. (1988). Streptococcus mutans endocarditis: report of three cases and review of the literature. Heart Lung 17, 209-212.

Verneuil, N., Sanguinetti, M., Le Breton, Y., Posteraro, B., Fadda, G., Auffray, Y., Hartke, A. \& Giard, J. C. (2004). Effects of the Enterococcus faecalis hypR gene encoding a new transcriptional regulator on oxidative stress response and intracellular survival within macrophages. Infect Immun 72, 4424-4431.

Yin, S., Daum, R. S. \& Boyle-Vavra, S. (2006). VraSR two-component regulatory system and its role in induction of $p b p 2$ and $v r a S R$ expression by cell wall antimicrobials in Staphylococcus aureus. Antimicrob Agents Chemother 50, 336-343.

Zhou, L., Lei, X. H., Bochner, B. R. \& Wanner, B. L. (2003). Phenotype microarray analysis of Escherichia coli K-12 mutants with deletions of all two-component systems. J Bacteriol 185, 4956-4972.

Edited by: M. Kilian 